\title{
The role of glucagon-like peptide-1 receptor agonists in the treatment of type 2 diabetes
}

\author{
Erin St Onge ${ }^{1}$, Shannon Miller ${ }^{1}$, Elizabeth Clements ${ }^{2}$, Lindsay Celauro ${ }^{2}$, \\ Ke'la Barnes ${ }^{2}$ \\ 'University of Florida, College of Pharmacy, Florida, USA; \\ ${ }^{2}$ Florida Hospital Celebration Health, Florida, USA
}

\section{ABSTRACT}

The role of GLP-1 agonists in the treatment of type 2 diabetes have been shown to be viable options for add-on therapy in diabetic patients, as well as potential monotherapy options. With six available GLP-1 agents, and new combination products in the pipeline, they are a promising drug class for type 2 diabetic patients, especially due to their extended dosing interval and potential weight loss benefits.

Key words: type 2 diabetes, GLP-1 receptor agonist, insulin, A1c, glucose, incretin, GLP-1RA

\section{INTRODUCTION}

An estimated 29.1 million Americans were living with diabetes in 2012, with more than one million diagnosed every year. ${ }^{[1]}$ The average medical expense for patients diagnosed with diabetes is approximately 2.3 times higher than those without diabetes. In 2012 alone, an estimated 245 billion healthcare dollars were spent on patients with diabetes. ${ }^{[1]}$ Type 2 diabetes (T2DM) accounts for 90 to $95 \%$ of all cases of diabetes, and is characterized by insulin resistance, hepatic gluconeogenesis, and progressive $\beta$-cell exhaustion or failure. ${ }^{[2,3]}$ The majority of T2DM patients are overweight, obese, or have an increased percentage of central adiposity. ${ }^{[2]}$ Patients may not exhibit the cardinal symptoms, such as polyuria and polydipsia, in the earlier stages, thus, approximately one out of four cases of diabetes remain undiagnosed. ${ }^{[2]}$ All patients with T2DM, regardless of diagnosis, are at an increased risk of developing diabetic complications. ${ }^{[2]}$ With these astounding statistics, it is clear why diagnosis and appropriate treatment of diabetes is imperative.

The initial recommendation for pharmacological treatment in T2DM patients differs between the American Academy of Clinical Endocrinologists/
American College of Endocrinology (AACE/ACE) and American Diabetes Association (ADA). While the ADA guidelines recommend initial monotherapy with metformin, AACE/ACE recommends any of the following as initial monotherapy: metformin, glucagon-like peptide-1 receptor agonists (GLP-1RA), sodium-glucose cotransporter 2 inhibitors (SGLT-2i), dipeptidyl peptidase-4 inhibitors (DPP4i), thiazolidinediones (TZD), alphaglucosidase inhibitors (AGI), sulfonylureas/ glinides (SU/GLN) ${ }^{[2,4]}$ Characteristics such as cost, adverse effects, and comorbidities, should be taken into account when choosing a medication. ${ }^{[2]}$ The ADA recommends consideration of insulin with or without additional agents in symptomatic patients or in those with elevated glucose or hemoglobin A1c (A1c) levels. ${ }^{[2]}$ Although AACE/ACE recommends monotherapy with any of the above agents, their guidelines utilize the Glucose Control Algorithm, a hierarchy of agents listed in order of recommendation strength. ${ }^{[4]}$ Metformin is listed first due to its favorable tolerability, low cost, and possible cardiovascular benefits. GLP-1RAs are listed second and may be a good option for initial monotherapy in patients in which metformin is contraindicated. GLP-1RAs have pronounced A1c lowering and are associated with weight loss due to their novel ability to enhance the incretin effect. ${ }^{[4]}$ 
The incretin effect is a phenomenon that occurs during glucose metabolism leading to 50 to $70 \%$ insulin secretion following ingestion of oral glucose. This same effect is not seen following intravenous glucose administration. ${ }^{[5]}$ Glucagon-like peptide-1 (GLP-1) and glucose-dependent insulinotropic polypeptide are two incretin hormones. When functioning properly, these hormones are anticipated to stimulate insulin secretion, decrease appetite, delay gastric emptying, and inhibit glucagon secretion, thus maintaining postprandial euglycemia. ${ }^{[6]}$ Dysregulation in these hormones may lead to postprandial hyperglycemia. ${ }^{[5]}$

Currently, there are two classes of incretin-based T2DM treatments available: GLP-1RAs and DPP-4i. GLP1RAs, which include albiglutide, dulaglutide, exenatide, exenatide extended-release, liraglutide, and lixisenatide, work by mimicking the glucoregulatory effects of GLP-1 and suppressing glucagon release, thereby enhancing glucose-dependent insulin secretion. ${ }^{[7]}$ Based on the aforementioned mechanism of the hormone GLP-1, medications in this class lead to weight loss, improved postprandial glucose (PPG) levels, and limited hypoglycemic events. ${ }^{[2]}$

GLP-1RAs are a relatively new class of T2DM medications, with the first being approved in the United States in 2005, and the most recent approved in July 2016. There are currently six agents approved within this class in the United States, and all available agents are formulated as subcutaneous injections with varying doses and frequencies. All GLP-1RAs are associated with nausea, which is expected to improve over the first few weeks of treatment. Additionally, although they do not all require renal dose adjustment, they do carry warnings for the risk of worsening renal failure, in addition to the rare occurrence of pancreatitis.

Table 1 compares the GLP-1RAs currently approved for use in the United States. ${ }^{[8-13]}$ These items are important to take into consideration when choosing an agent. Ultimately, the choice of GLP-1RA should be based on tolerability, cost, ease of use, and efficacy.

\section{EVIDENCE FOR USE OF GLP-1 AGONISTS WITH ORAL AGENTS}

Each of the GLP-1RAs have been used in combination with various oral therapies including metformin, sulfonylureas, and TZDs. This section will review the evidence available regarding the use of GLP-1RAs in combination with monotherapy, dual therapy, or triple therapy with various oral antidiabetic agents.

\section{Exenatide}

The combination of exenatide with metformin monotherapy has been examined in several studies. In one study, 336 patients on maximal doses of metformin were randomized to receive exenatide or placebo after a 4-week placebo run-in period for a total of 30 weeks. ${ }^{[14]} \mathrm{A}$ dosedependent reduction in A1c was found in patients taking exenatide versus the placebo $(P<0.001)$. Weight loss was evident in patients taking exenatide, especially those with a baseline body mass index (BMI) $\geq 30 \mathrm{~kg} / \mathrm{m}^{2}(P \leq 0.05$ versus placebo). Two studies looked at the combination of exenatide and metformin compared to the addition of a sulfonylurea. ${ }^{[15,16]}$ In one study, 128 patients on metformin were randomized to receive exenatide $5 \mathrm{mcg}$ twice daily (titrated to $10 \mathrm{mcg}$ twice daily) or glibenclamide $2.5 \mathrm{mg}$ three times daily (titrated to $5 \mathrm{mg}$ three times daily) for 52 weeks. $^{[15]}$ Similar improvements in A1c, fasting plasma glucose (FPG), and PPG were found in both groups; however, body weight and BMI significantly improved in patients taking exenatide compared to glibenclamide. A similar study in 111 patients examined the effect of adding exenatide versus glimepiride to metformin treated patients for 52 weeks. ${ }^{[16]}$ Results of this study revealed similar improvements in glycemic control between groups with a significant reduction in BMI in patients on exenatide compared to glimepiride $(P<0.05)$. Exenatide added to monotherapy with metformin has also been compared to premixed insulin. ${ }^{[17]}$ In this study, 363 patients were randomized to receive exenatide 5 mcg twice daily for 4 weeks titrated to $10 \mathrm{mcg}$ twice daily or premixed insulin aspart for 26 weeks. Results of this study showed exenatide was non-inferior to premixed insulin aspart in reducing A1c and was associated with a lower risk of hypoglycemia $(P<0.05)$. In addition, weight decreased significantly in the exenatide group compared to the patients taking premixed insulin $(P<0.001)$.

Several studies have examined the effect of exenatide in combination with metformin with or without a sulfonylurea. ${ }^{[18-21]}$ A 30 -week study randomized 733 patients currently taking metformin plus a sulfonylurea to exenatide 5 mcg twice daily, exenatide $10 \mathrm{mcg}$ twice daily, or placebo. ${ }^{[18]}$ Use of both doses of exenatide resulted in significant improvements in A1c compared to placebo $(P<0.0001)$. In addition, both doses of exenatide resulted in significant weight loss compared to placebo. Rates of hypoglycemia were higher with exenatide use ( $P$ value not reported). Another study of 551 patients compared the addition of exenatide versus insulin glargine to patients uncontrolled on metformin and sulfonylurea combination therapy for 26 weeks. ${ }^{[19]}$ In this study, patients received either exenatide $10 \mathrm{mcg}$ twice daily or insulin glargine once daily titrated to a fasting glucose $<100 \mathrm{mg} / \mathrm{dL}$. Similar reductions in 


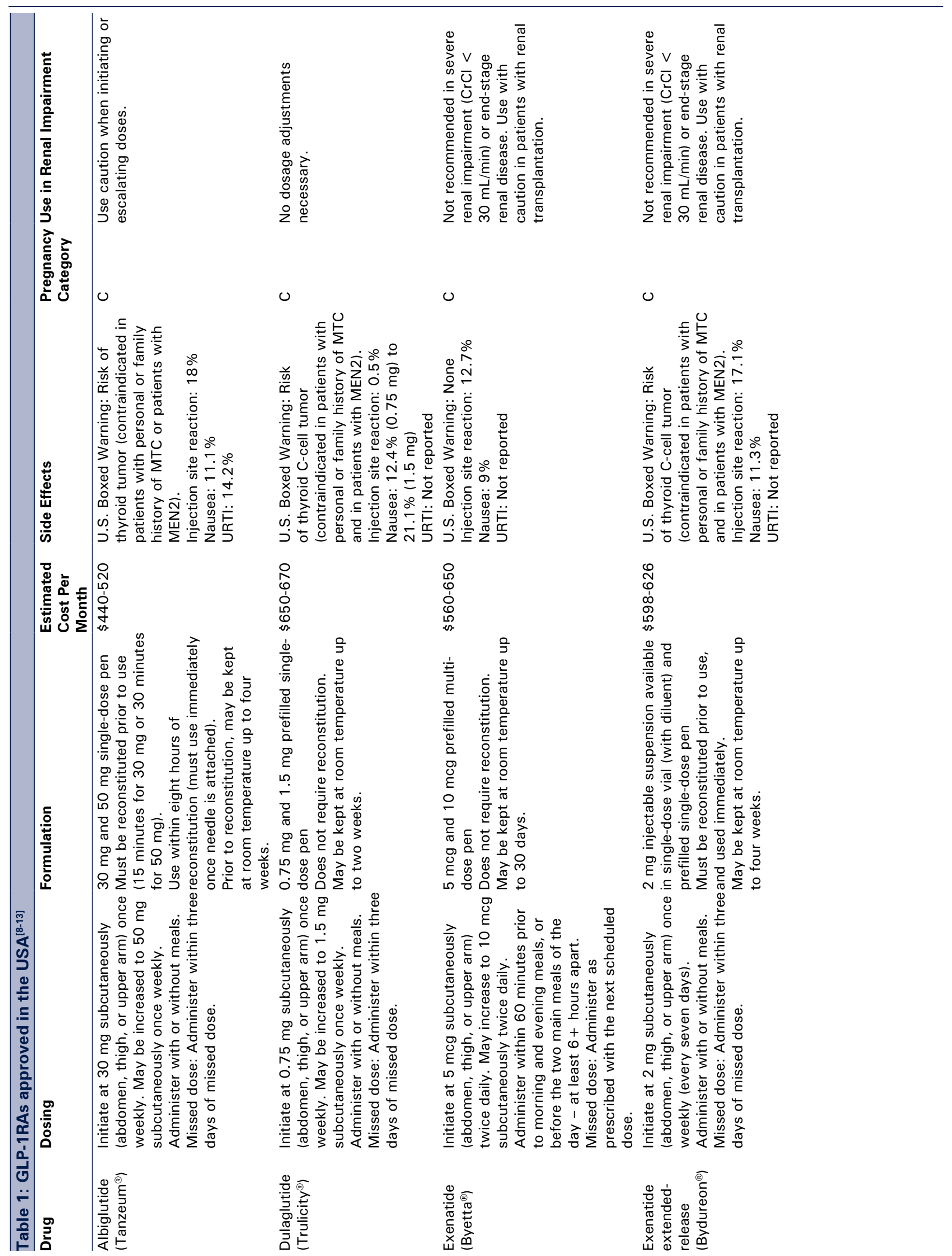




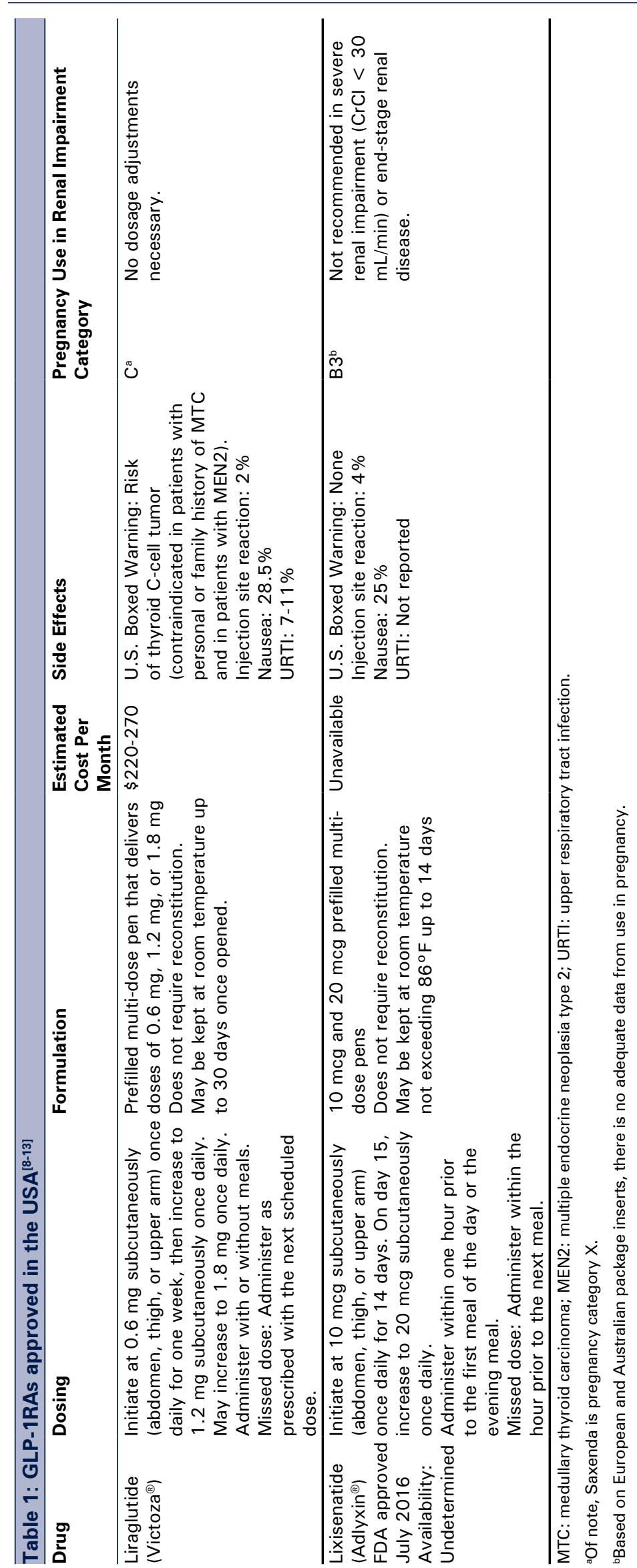


A1c were seen with both interventions; however, insulin glargine improved FPG to a greater extent. Body weight decreased significantly in patients receiving exenatide while hypoglycemia rates were similar between groups. The addition of exenatide to patients uncontrolled on metformin and a sulfonylurea has also been compared to the addition of biphasic insulin aspart in a 52-week trial involving 501 patients. $^{[20]}$ Results of this study showed exenatide to be non-inferior to biphasic insulin aspart in improving glycemic control. The use of exenatide resulted in significant reductions in body weight, while patients receiving insulin aspart gained weight.

A study examining the effect of adding exenatide or placebo to patients currently taking metformin and/or a sulfonylurea was conducted over 24 weeks. ${ }^{[22]}$ In this study, 194 overweight or obese patients were randomized to receive exenatide $10 \mathrm{mcg}$ twice daily or placebo. Results of this study showed significant improvements in weight $(P=0.003)$ and A1c $(P$ $<0.0001)$, as well as systolic $(P<0.001)$ and diastolic blood pressure $(P=0.04)$. The addition of exenatide compared to placebo added to sulfonylurea monotherapy was investigated in a study of 377 patients. ${ }^{[23]}$ Patients were randomized to receive exenatide $5 \mathrm{mcg}$ twice daily, exenatide $10 \mathrm{mcg}$ twice daily, or placebo for 30 weeks. Significant reductions in A1c were evident in both exenatide groups compared to placebo $(P<0.001)$. Weight loss was evident in both exenatide groups; however, only patients in the $10 \mathrm{mcg}$ twice daily group experienced a significant reduction in weight when compared to placebo $(P<0.05)$. The addition of exenatide compared to insulin glargine was examined in 235 patients uncontrolled on two (metformin + sulfonylurea) or three (metformin + sulfonylurea + TZD) oral medications. ${ }^{[21]}$ Patients were randomized to receive exenatide 5 to $10 \mathrm{mcg}$ twice daily or titrated insulin glargine once daily for 26 weeks. Exenatide and insulin glargine had similar effects on A1c; however, improvements in body weight were significantly better with exenatide than glargine $(P<0.001)$. Hypoglycemia rates were similar between groups with the exception of nocturnal hypoglycemia. A 26-week study comparing the addition of exenatide or placebo to patients currently taking a TZD with or without metformin was conducted in 165 patients. ${ }^{[2]}$ As expected, A1c reductions were significantly greater in patients treated with exenatide compared to placebo $(P<0.001)$. Surprisingly, the mean reduction in body weight was similar in both the exenatide and placebo groups at study endpoint. Rates of nausea and vomiting were significantly greater with exenatide treatment, while the incidence of hypoglycemia was similar between groups.

\section{Liraglutide}

The concomitant use of liraglutide with oral agents such as metformin, TZDs, and sulfonylureas as monotherapy or in combination has been studied in several trials. The addition of liraglutide compared to sitagliptin in patients uncontrolled on metformin monotherapy was examined in a study of 665 patients. ${ }^{[25]}$ In this study, the patients were randomized to receive liraglutide $1.2 \mathrm{mg}$ daily, liraglutide $1.8 \mathrm{mg}$ daily or sitagliptin $100 \mathrm{mg}$ daily for 26 weeks. Mean A1c was significantly reduced in both liraglutide groups compared to sitagliptin ( $P<0.0001$ for both doses). Weight loss was also significantly greater with liraglutide compared to sitagliptin $(P<0.0001$ for both doses). Liraglutide compared to placebo added to metformin + TZD was examined in another study. ${ }^{[2]}$ Similar to the previous study, patients were randomized to receive liraglutide 1.2 $\mathrm{mg}$ daily, liraglutide $1.8 \mathrm{mg}$ daily, or placebo for 26 weeks. Results of this study showed a significant decrease in A1c in both liraglutide groups compared to placebo. Patients experienced weight loss with liraglutide therapy while weight increased in the placebo treated patients $(P<$ $0.0001)$. Minor hypoglycemia was more common in patients treated with liraglutide; however, there were no cases of serious hypoglycemia reported. Another study compared the effect of adding liraglutide, insulin glargine, or placebo to patients currently taking metformin plus a sulfonylurea. ${ }^{[27]}$ In this 26-week trial, 581 patients were randomized to receive liraglutide $1.8 \mathrm{mg}$ daily, insulin glargine once daily, or placebo. After 26 weeks, A1c decreased significantly in patients using liraglutide compared to placebo $(P<0.0001)$ as well as insulin glargine $(P=0.0015)$. Body weight was also significantly improved in patients using liraglutide compared to placebo $(P=0.0001)$ and insulin glargine $(P$ $<0.0001)$. Hypoglycemia rates were similar between the liraglutide and insulin glargine groups; hypoglycemia was much less common in patients receiving placebo. The addition of liraglutide compared to a TZD has been studied in 1041 patients taking sulfonylurea monotherapy. ${ }^{[28]}$ In this study, patients were randomized to receive liraglutide $0.6 \mathrm{mg}$ daily, liraglutide $1.2 \mathrm{mg}$ daily, liraglutide $1.8 \mathrm{mg}$ daily, rosiglitazone $4 \mathrm{mg}$ daily, or a placebo for 26 weeks. A1c reductions were greater with liraglutide $1.2 \mathrm{mg}$ and $1.8 \mathrm{mg}$ daily, but not with liraglutide $0.6 \mathrm{mg}$, compared to rosiglitazone or placebo $(P<0.0001$ for all comparisons). Body weight with liraglutide use did not change significantly compared to placebo; however, body weight significantly improved between liraglutide and rosiglitazone $(P<0.0001)$.

\section{Albiglutide}

A 2-year trial examined the effect of adding albiglutide versus sitagliptin, glimepiride, or placebo to patients with T2DM uncontrolled on metformin monotherapy. ${ }^{[29]}$ In this study, 1012 patients were randomized to receive albiglutide $30 \mathrm{mg}$ weekly, sitagliptin $100 \mathrm{mg}$ daily, glimepiride $2 \mathrm{mg}$ daily, or a placebo. Albiglutide significantly reduced the A1c compared to placebo, sitagliptin, and glimepiride $(P<0.0001, P=0.0001$, and $P=0.0033$, respectively $)$. 
Changes in weight were significant for the comparison of albiglutide and glimepiride only $(P<0.0001)$. The rate of serious adverse events was similar between the albiglutide and comparison groups. The addition of albiglutide versus pioglitazone to patients uncontrolled on metformin and glimepiride was evaluated in a 52-week study. ${ }^{[30]}$ Patients were randomized to receive albiglutide 30 to $50 \mathrm{mg}$ once weekly, pioglitazone 30 to $45 \mathrm{mg}$ once daily, or a placebo. At the end of the study, albiglutide decreased the A1c significantly compared to placebo $(P<0.0001)$ and was shown to be non-inferior to treatment with pioglitazone in lowering the A1c $(P=0.27)$. Changes in body weight were significantly greater with albiglutide compared to pioglitazone $(P<0.0001)$. Another study evaluated the safety and efficacy of adding albiglutide once weekly compared to daily insulin glargine to patients currently uncontrolled on metformin with or without a sulfonylurea for one year. ${ }^{[31]}$ Patients were randomized to receive albiglutide 30 to $50 \mathrm{mg}$ once weekly or titrated daily insulin glargine. Results of this study showed albiglutide was non-inferior to insulin glargine in A1c reduction. Changes in body weight as well as symptomatic hypoglycemia favored the use of albiglutide over insulin glargine.

\section{Dulaglutide}

Two studies examined the effect of adding dulaglutide to patients uncontrolled on metformin monotherapy. ${ }^{[32,33]}$ In one study, 1098 patients were randomized to receive dulaglutide $1.5 \mathrm{mg}$ weekly, dulaglutide $0.75 \mathrm{mg}$ weekly, sitagliptin $100 \mathrm{mg}$ daily, or placebo for 52 weeks. ${ }^{[32]}$ Results of this study showed dulaglutide to be superior to sitagliptin in lowering A1c as well as improving body weight ( $P<0.001$ for both comparisons). In addition, no severe hypoglycemia events were reported in any group. A study by Dungan et al. compared the addition of dulaglutide $1.5 \mathrm{mg}$ weekly to liraglutide $1.8 \mathrm{mg}$ daily in 599 patients on metformin monotherapy for 26 weeks. ${ }^{[33]}$ The results of this study showed dulaglutide was non-inferior to liraglutide in reducing A1c. The most common adverse events were gastrointestinal in nature for both groups and no severe hypoglycemia was reported in either group. Dulaglutide was also compared to the addition of exenatide or placebo in patients currently being treated with metformin + pioglitazone ${ }^{[34]}$ In this study, patients were randomized to receive dulaglutide $1.5 \mathrm{mg}$ weekly, dulaglutide $0.75 \mathrm{mg}$ weekly, exenatide $10 \mathrm{mcg}$ twice daily, or a placebo. Both doses of dulaglutide were shown to be superior to placebo as well as exenatide in lowering A1c $(P<0.001$ for all comparisons). Hypoglycemia rates were lower with dulaglutide than exenatide at all time periods and no patients in the dulaglutide groups reported severe hypoglycemia.

\section{Lixisenatide}

Lixisenatide, the newest GLP-1 agonist to be approved in the US, has been studied in patients on metformin monotherapy in two studies. ${ }^{[35,36]}$ In the first study, 680 patients were randomized to receive lixisenatide $20 \mathrm{mcg}$ in the evening, lixisenatide $20 \mathrm{mcg}$ in the morning, placebo in the evening, or placebo in the morning. ${ }^{[35]}$ Both lixisenatide groups significantly reduced A1c compared to placebo $(P<0.0001)$. Body weight decreased similarly amongst all groups. Nausea and vomiting were reported more often with lixisenatide; no serious episodes of hypoglycemia were reported. The other study examined the safety and efficacy of adding lixisenatide versus exenatide to patients on current metformin therapy. ${ }^{[36]}$ In this study, 634 patients were randomized to receive lixisenatide $20 \mathrm{mcg}$ once daily or exenatide $10 \mathrm{mcg}$ twice daily for 24 weeks. Lixisenatide use was shown to be non-inferior to exenatide in A1c reduction. Weight loss was similar between groups as was the incidence of adverse events (including serious adverse events). A study examining the effect of adding lixisenatide to patients uncontrolled on a sulfonylurea with or without metformin was conducted by Rosenstock et al. ${ }^{\left[{ }^{37]}\right.}$ In this study, 859 patients were randomized to receive lixisenatide $20 \mathrm{mcg}$ daily or placebo for 24 weeks. A1c was significantly reduced by lixisenatide use $(P<0.0001$, versus placebo). Body weight was significantly reduced by lixisenatide and symptomatic hypoglycemia was similar between groups. The addition of lixisenatide to patients uncontrolled on pioglitazone with or without metformin was studied in 484 patients. ${ }^{[38]}$ Patients were randomized to receive lixisenatide 20 mcg once daily or placebo for 24 weeks. Lixisenatide use produced significant improvements in A1c compared to placebo $(P<0.0001)$. Body weight decreased slightly with lixisenatide use; however, there were no significant differences between groups. Symptomatic hypoglycemia rates were similar between groups with no severe episodes reported.

\section{REVIEW OF EVIDENCE FOR USE OF GLP-1 AGONISTS WITH BASAL INSULIN}

Based on the mechanism of GLP-1RAs, their use in combination with basal insulin has been an interest of research. Basal insulin targets FBG while GLP-1RAs primarily focus on reducing post-prandial glycemic excursions. Evidence suggests this complementary approach reduces insulin dose requirements, subsequent weight gain, and enables improved control of A1c. ${ }^{[3,40,41]}$ In addition, GLP-1RAs are less likely to cause hypoglycemia, which affords an attractive option when compared with bolus insulin. The following will review the combination of GLP-1RAs with basal insulin, as well as premixed and 
prandial formulations. A more comprehensive list of trials can be found in Table 2 .

Several studies have evaluated the combination of GLP1RAs with basal insulin. A prospective, randomized, placebo-controlled trial assessed the glycemic control when twice daily exenatide was added to insulin glargine ( + oral antidiabetic drugs) in 261 participants. ${ }^{[42]}$ Average insulin dose increased in both groups, however the placebo groups required more insulin (20 units/day versus 13 units/day). A1c decreased significantly from baseline in the exenatide group compared to placebo $(P<0.001)$. Incretin-based therapies look exceptionally effective in Asian patients who tend to have insulin deficiency and evidence of underlying GLP-1 insufficiency. ${ }^{[43,44]}$ The efficacy and safety of lixisenatide versus placebo was evaluated in 311 Asian subjects. ${ }^{[43]}$ Adding once daily lixisenatide to basal inulin ( + a sulfonylurea) significantly improved two-hour PPG and allowed more patients to achieve an A1c $<7 \%$ when compared to placebo. More patients in the lixisenatide group as compared to placebo experienced gastrointestinal events, with nausea/vomiting being the most commonly reported adverse effect (39.6\% vs. $18.2 \%$, respectively). Riddle et al. conducted a 24 -week double blind, parallel group trial studying 446 patients inadequately controlled on metformin and insulin glargine who were randomized to the addition of lixisenatide or placebo. ${ }^{[45]}$ Significant reductions in A1c, PPG, and body weight were noted in the lixisenatide group. Nausea, vomiting, and symptomatic hypoglycemia were more prominent in the lixisenatide group.

The use of a GLP-1RA in combination with various insulin types was evaluated in a retrospective review of 188 patients. ${ }^{[4]}$ Eligible patients had a diagnosis of T2DM and must have received twice daily exenatide in addition to basal insulin, basal and prandial insulin, or prandial insulin only. Adding exenatide reduced A1c and weight significantly at each time period reported and remained at 27 months. Approximately $70 \%$ of patients had a diagnosis of diabetes for greater than 10 years, highlighting an improvement in glycemic control even in advanced stages of the disease. Lind and colleagues conducted a study evaluating the addition of liraglutide or twice daily exenatide to various insulin regimens (basal only, multiple daily injections, or premixed). ${ }^{[4]}$ The addition of the GLP-1RAs were associated with improved glycemic control $(-1.2 \%$ A1c, $P<0.001)$, decreased weight $(-7.1 \mathrm{~kg}, P<0.001)$ and reduced total insulin dose $(-38.9$ units, $P<0.001)$. In obese patients on high dose U-500 insulin (192 units), the addition of liraglutide also produced a reduction in A1c, weight, and a decrease in insulin dose by approximately $28 \% .{ }^{[48]}$

Several studies have reviewed the benefits of either adding GLP-1RAs or prandial insulin to patients not controlled on basal insulin. ${ }^{[49-52]}$ Patients taking basal insulin (+ oral agents) were randomized to weekly albiglutide $(n=282)$ or three times daily prandial lispro $(n=281) \cdot{ }^{[49]}$ After 26 weeks, A1c decreased from baseline by $0.82 \%$ in the albiglutide arm and $0.66 \%$ in the lispro group. $(P<0.001)$. Weight decreased from baseline for albiglutide by $0.7 \mathrm{~kg}$, but increased in the lispro group by $0.8 \mathrm{~kg}$ $(P<0.0001)$. As expected, lower rates of hypoglycemia and more gastrointestinal side effects were observed in the albiglutide group compared to the lispro group. A similarly designed study was conducted over 30 weeks randomizing 627 patients to twice daily exenatide or thrice-daily mealtime lispro added to insulin glargine and metformin. ${ }^{[50]}$ Mean A1c changes were non-inferior for exenatide compared with lispro $(-1.13$ and $-1.10 \%$, respectively). The exenatide group noted a lower FBG and greater decrease in weight. A greater number of patients experienced treatment related adverse events in the exenatide group, but interestingly reported more treatment satisfaction and better quality of life.

Fixed dose combinations of GLP-1RAs and basal insulin have also been studied. ${ }^{[3-56]}$ The addition of liraglutide to basal insulin was studied in $413 \mathrm{~T} 2 \mathrm{DM}$ patients in a randomized, parallel, 26-week study. ${ }^{[54]}$ In this trial, a fixed dose combination of liraglutide plus insulin degludec was compared to degludec alone in patients currently taking metformin with or without sulfonylureas/glinides. The A1c decreased by $1.9 \%$ in the combination insulin degludec/ liraglutide group compared to $0.9 \%$ with degludec alone $(P<0.001)$. Body weight decreased significantly in the degludec/liraglutide arm with no weight change in the insulin degludec arm. Adverse events were similar between the groups. A pooled analysis of five other studies using insulin degludec plus liraglutide also demonstrated the safety and efficacy of the combination. ${ }^{[5]}$ Another fixedratio combination of a GLP-1RA and basal insulin was performed in 323 participants with a mean age of 57 years and T2DM for six to seven years. ${ }^{[56]}$ The combination of lixisenatide and insulin glargine was compared to insulin glargine in insulin-naïve participants currently on metformin. The mean A1c was decreased by $1.7 \%$ in the combination lixisenatide/insulin glargine group and $1.5 \%$ in the insulin glargine arm $(P=0.01)$. Body weight decreased in the combination group $(1 \mathrm{~kg})$ and increased in the insulin only arm $(0.5 \mathrm{~kg})(P<0.0001)$; no increase in hypoglycemia was noted.

Reviewed studies highlight several patient populations that may benefit from the addition of a GLP-1RA. Adding a GLP-1RA compared to intensive bolus insulin appears equally efficacious, with lower hypoglycemia potential and decreased number of injections. In addition, the patients on large doses of insulin who remain unable 


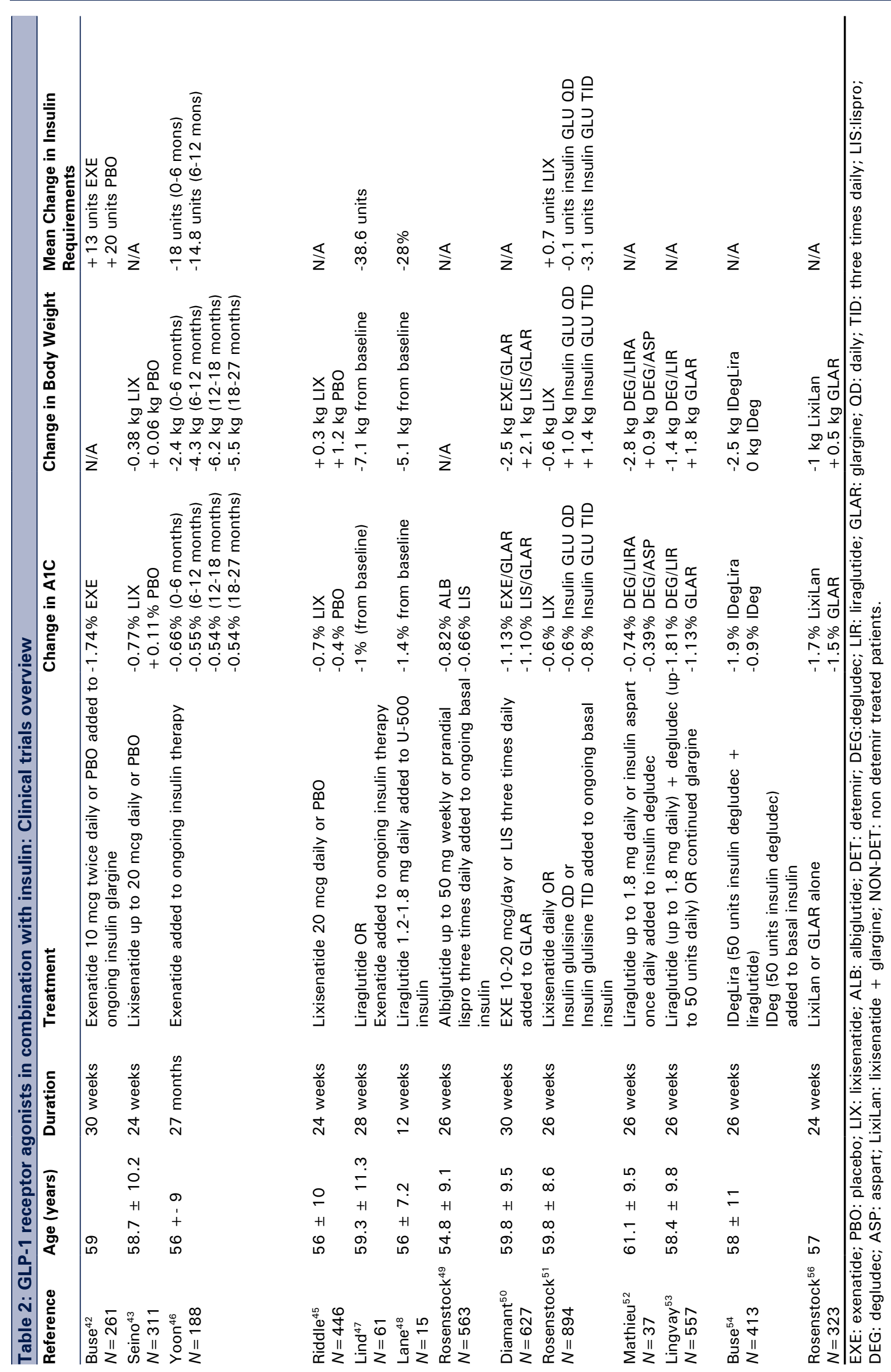


to meet glycemic goals may benefit from this class by lowering the required insulin dose which is expected to lead to subsequent weight reduction. Overweight patients, regardless of glycemic control, will likely find GLP-1RA agonists beneficial, as well as patients with notable postprandial hyperglycemia.

\section{FUTURE DIRECTIONS}

According to both the AACE/ACE and ADA guidelines, if the glycemic goal remains unmet with titrated basal insulin, the addition of a GLP-1RA (or SGLT-2i or DPP$4 i)$ is a reasonable choice for intensifying post-prandial control. ${ }^{[2,4]}$ Currently, there are two GLP-1RA and basal insulin combination products, insulin degludec/liraglutide combination (IDegLira ${ }^{\circledR}$ ) and insulin glargine/lixisenatide (LixiLan $\left.{ }^{\circledR}\right)$, in the pipeline.

The FDA is anticipated to make a decision regarding the approval of insulin degludec/liraglutide by December 2016. ${ }^{[57]}$ As previously mentioned, Lingvay et al. conducted a phase 3 randomized control trial to assess the reduction in $\mathrm{A} 1 \mathrm{c}$ and found insulin degludec/liraglutide compared to insulin glargine were non-inferior to each other. ${ }^{[53]}$ Another phase 3 clinical trial involved 1663 patients randomized to receive either insulin degludec, liraglutide, or insulin degludec/ liraglutide combination for 12 weeks. ${ }^{[58]}$ Statistically, more patients were able to achieve an A1c $<7 \%$ in the insulin degludec/liraglutide combination group $(n=77)$ compared to either insulin degludec $(n=55)$ or liraglutide $(n=60)$ alone $(P<0.0001)$. Insulin degludec/liraglutide combination averaged a larger change in FBG values from baseline compared to either insulin degludec or liraglutide alone $(P<0.0001)$.

Insulin glargine/lixisenatide combination is currently pending approval by the FDA. It is anticipated that this combination will be less costly than insulin degludec/ liraglutide. In the study conducted by Rosenstock et al., significant A1c reductions were found in the combination group compared with insulin glargine alone. ${ }^{[56]}$ In addition, these patients experienced greater weight loss, no change in hypoglycemia risk, and fewer gastrointestinal adverse effects.

A majority of the currently available GLP-1RAs are dosed once weekly, which may aid in improved adherence. The newest agent in the pipeline, efpeglenatide (Exendin $\left.{ }^{\circledR}\right)$, may be dosed monthly. ${ }^{[59]}$ A phase 1B trial compared weekly efpeglenatide, monthly efpeglenatide, liraglutide daily, and placebo, on insulin secretion rates. Weekly and monthly efpeglenatide had greater insulin secretion than both daily liraglutide and placebo. While results look promising, further clinical trials are expected to occur in late 2016. ${ }^{[60]}$

\section{CONCLUSION}

T2DM is a complex disease which often requires multiple interventions for glycemic control. GLP-1RAs have been shown to be viable options for add-on therapy to patients on oral medications as well as basal insulin. With six GLP1RAs available, each with its own unique characteristics, clinicians are able to choose an agent based on patient characteristics and preferences. Combination products, containing a GLP-1RA and basal insulin, will soon be available. Combining these two agents not only targets the pathophysiology of diabetes from different mechanisms, but also has the added benefit of improved adherence and potential weight loss.

\section{Conflict of Interest}

The authors have to conflict of interest to declare.

\section{REFERENCES}

1. American Diabetes Association. Statistics about diabetes (April 2016). Available at: http://www.diabetes.org/diabetes-basics/statistics. Accessed on October 17, 2016.

2. American Diabetes Association. Standards of medical care in diabetes - 2016. Diabetes Care 2016;39:S1-112.

3. Jellinger, PS. Focus on Incretin-Based Therapies: Targeting the Core Defects of Type 2 Diabetes. Postgrad Med 2011;121:53-65.

4. Consensus statement by the American Association of Clinical Endocrinologists and American College of Endocrinology on the comprehensive type 2 diabetes management algorithm - 2016 executive summary. Endocr Pract 2016;22;84-113.

5. Vilsbøll T, Holst JJ. Incretins, insulin secretion and type 2 diabetes mellitus. Diabetologia 2004;47:357-66.

6. Nauck MA. Unraveling the science of incretin biology. Am J Med 2009;122:S3-10.

7. Tibaldi JM. Incorporating incretin-based therapies into clinical practice for patients with type 2 diabetes. Adv Ther 2014;31:289-317.

8. Tanzeum (albiglutide) package insert. Wilmington, DE: GlaxoSmithKline; 2016 Sept.

9. Trulicity (dulaglutide) package insert. Indianapolis, IN: Eli Lilly; 2015 March

10. Bydureon (exenatide extended-release) package insert. Wilmington, DE: AstraZeneca; 2015 Sept.

11. Byetta (exenatide) package insert. San Diego, CA: Amylin Pharmaceuticals; 2009 Oct.

12. Victoza (liraglutide) package insert. Princeton, NJ: Novo Nordisk; 2010 Jan.

13. Lyxumia (lixisenatide) package insert. Macquarie Park NSW, Australia: Sanofi-Aventis; 2013 April

14. DeFronzo R, Kim D, Ratner R, Kim DD, Fineman MS, Baron AD. Effects of exenatide (exendin-4) on glycemic control and weight over 30 weeks in metformin-treated patients with type 2 diabetes. Diabetes Care 2005; 28: $1092-100$.

15. Derosa G, Maffioli P, Salvadeo SAT, Ferrari I, Ragonesi PD, Querci F, et al. Exenatide versus glibenclamide in patients with diabetes. Diabetes Technol Therapeut 2010; 12: 233-40.

16. Derosa G, Putignano P, Bossi AC, Bonaventura A, Quercid F, Franzettie IG, et al. Exenatide or glimepiride added to metformin on metabolic control and on insulin resistance in type 2 diabetic patients. Eur J Pharmacol 2011; 666: 251-6. 
17. Gallwitz B, Böhmer M, Segiet T, Mölle A, Milek K, Becker B, et al. Exenatide twice daily versus premixed insulin aspart 70/30 in metformintreated patients with type 2 diabetes. Diabetes Care 2011; 34: 604-6.

18. Kendall D, Riddle MC, Rosenstock J, Zhuang D, Kim D, Fineman MS, et al. Effects of exenatide (exendin-4) on glycemic control over 30 weeks in patients with type 2 diabetes treated with metformin and a sulfonylurea. Diabetes Care 2005; 28: 1083-91.

19. Heine R, VanGaal L, Johns D, Mihm MJ, Widel MH, Brodows RG, et al. Exenatide versus insulin glargine in patients with suboptimally controlled type 2 diabetes. Annals of Internal Medicine 2005; 143: 559-69.

20. Nauck M, Duran S, Kim D, Johns D, Northrup J, Festa A, et al. A comparison of twice-daily exenatide and biphasic insulin aspart in patients with type 2 diabetes who were suboptimally controlled with sulfonylurea and metformin: a non-inferiority study. Diabetologia 2007; 50: 259-67.

21. Davies MJ, Donnelly R, Barnett AH, Jones S, Nicolay C, Kilcoyne A, et al. Exenatide compared with long-acting insulin to achieve glycemic control with minimal weight gain in patients with type 2 diabetes: results of the Helping Evaluate Exenatide in patients with diabetes compared with LongActing insulin (HEELA) study. Diabetes Obes Metab 2009; 11: 1153-62.

22. Apovian CM, Bergenstal RM, Cuddihy RM, Qu Y, Lenox S, Lewis MS, et al. Effects of exenatide combined with lifestyle modification in patients with type 2 diabetes. Am J Med 2010; 123: e9-17.

23. Buse JB, Henry RR, Han J, Kim DD, Fineman M, Baron AD, et al. Effects of exenatide (exendin-4) on glycemic control over 30 weeks in sulfonylurea-treated patients with type 2 diabetes. Diabetes Care 2004; 27: 2628-35.

24. Liutkus J, Guzman JR, Norwood P, Pop L, Northrup J, Cao D, et al. A placebo-controlled trial of exenatide twice-daily added to thiazolidinediones alone or in combination with metformin. Diabetes Obes Metab 2010; 12: 1058-65.

25. Pratley R, Nauck M, Bailey T, Montanya E, Cuddihy R, Filetti S, et al. One year of liraglutide treatment offers sustained and more effective glycaemic control and weight reduction compared with sitagliptin, both in combination with metformin, in patients with type 2 diabetes: a randomized, parallel-group, open-label trial. Int J Clin Pract 2011; 65: 397-407.

26. Zinman B, Gerich J, Buses JB, Lewin A, Schwartz S, Raskin P, et al. Efficacy and safety of the human glucagon-like peptide- 1 analog liraglutide in combination with metformin and thiazolidinedione in patients with type 2 diabetes (LEAD-4 Met + TZD). Diabetes Care 2009; 32: 1224-30.

27. Russell-Jones D, Vaag A, Schmitz O, Sethi BK, Lalic N, Antic S, et al. Liraglutide vs insulin glargine and placebo in combination with metformin and sulfonylurea therapy in type 2 diabetes mellitus (LEAD- 5 met+SU): a randomized controlled trial. Diabetologia 2009; 52: 2046-55.

28. Marre M, Shaw J, Brandle M, Bebakar WMW, Kamaruddin NA, Strand J, et al. Liraglutide, a once-daily human GLP-1 analogue, added to a sulphonylurea over 26 weeks produces greater improvements in glycaemic and weight control compared with adding rosiglitazone or placebo in subjects with Type 2 diabetes (LEAD-1 SU). Diabetic Med 2009; 26: 268-78.

29. Ahren B, Johnson SL, Stewart M, Cirkel DT, Yang F, Perry C, et al. Harmony 3: 104-week randomized, double-blind, placebo- and activecontrolled trial assessing the efficacy and safety of albiglutide compared with placebo, sitagliptin, and glimepiride in patients with type 2 diabetes taking metformin. Diabetes Care 2014; 37: 2141-8.

30. Stewart M, Home P, Yang F, Perry C, Carr MC. 52-week efficacy of albiglutide vs placebo and vs pioglitazone in triple therapy (background metformin and glimepiride) in patients with type 2 diabetes: HARMONY 5 study. Diabetologia 2013; 56(Supp 1): S361.

31. Pratley R, Stewart M, Cirkel D, Ye J, Perry C, Carr MC. HARMONY 4: 52-week efficacy of albiglutide vs insulin glargine in patients with type 2 diabetes mellitus. Diabetologia 2013; 56(Supp 1): S360-1.

32. Nauck M, Weinstock RS, Umpierrez GE, Guerci B, Skrivanek Z, Milicevic Z. Efficacy and safety of dulaglutide versus sitagliptin after 52 weeks in type 2 diabetes in a randomized controlled trial (AWARD-5). Diabetes Care 2014; 37: 2149-58.
33. Dungan KM, Povedano ST, Forst T, González González JG, Atisso C, Sealls W, et al. Once-weekly dulaglutide versus once-daily liraglutide in metformin-treated patients with type 2 diabetes (AWARD-6): a randomized, open-label, phase 3, non-inferiority trial. Lancet 2014; 384: 1349-57.

34. Wysham C, Blevins T, Arakaki R, Colon G, Garcia P, Atisso C, et al. Efficacy and safety of dulaglutide added onto pioglitazone and metformin versus exenatide in type 2 diabetes in a randomized controlled trial (AWARD-1). Diabetes Care 2014; 37: 2159-67.

35. Ahren B, Dimas AL, Miossec P, Saubadu S, Aronson R. Efficacy and safety of lixisenatide once-daily morning or evening injections in type 2 diabetes inadequately controlled on metformin (GetGoal-M). Diabetes Care 2013; 36: 2543-50.

36. Rosenstock J, Raccah D, Koranyi L, Maffei L, Boka G, Miossec P, et al. Efficacy and safety of lixisenatide once daily versus exenatide twice daily in type 2 diabetes inadequately controlled on metformin: a 24-week, randomized, open-label, active-controlled study (GetGoal-X). Diabetes Care 2013; 36: 2945-51.

37. Rosenstock J, Hanefeld M, Shamanna P, Mind KW, Bokae G, Miossec $\mathrm{P}$, et al. Beneficial effects of once-daily lixisenatide on overall and postprandial glycemic levels without significant excess of hypoglycemia in type 2 diabetes inadequately controlled on a sulfonylurea with or without metformin (GetGoal-S). J Diabetes Complicat 2014; 28: 386-92.

38. Pinget M, Goldenberg R, Niemoeller E, Muehlen-Bartmer I, Guo H, Aronson R. Efficacy and Safety of lixisenatide once daily versus placebo in type 2 diabetes insufficiently controlled on pioglitazone (GetGoal-P). Diabetes Obes Metab 2013; 15: 1000-7.

39. Davies ML, Pharm DQ, Drab SR. GLP1-RA add-on therapy in patients with type 2 diabetes currently on a bolus containing insulin regimen. Pharmacotherapy 2016; 36: 893-905.

40. Vora J. Combining incretin-based therapies with insulin. Diabetes Care 2013; 36: S226-32.

41. Eng C, Kramer CK, Zinman B, Retnakaran R. Glucagon-like peptide-1 receptor agonist and basal insulin combination treatment for the management of type 2 diabetes: a systematic review and meta-analysis. The Lancet. 2014;384(9961):2228-34.

42. Buse JB, Bergenstal RM, Glass LC,Heilmann CR,Lewis MS, Kwan AYM, et al. Use of twice-daily exenatide in basal insulin-treated patients with type 2 diabetes. Ann of Int Med 2011; 154: 103-12.

43. Seino Y, Min KW, Niemoeller E, Takami A. Randomized, double-blind, placebo-controlled trial of the once-daily GLP-1 receptor agonist lixisenatide in Asian patients with type 2 diabetes insufficiently controlled on basal insulin with or without a sulfonylurea (GetGoal-L-Asia). Diabetes Obes Metab 2012; 14: 910-7.

44. Yabe D, Watanabe K, Sugawara K, Kuwata H, Kitamoto Y, Sugizaki K, et al. Comparison of incretin immunoassays with or without plasma extraction: incretin secretion in Japanese patients with type 2 diabetes. J Diabetes Invest 2012; 3: 70-9.

45. Riddle MC, Forst T, Aronson R, Sauque-Reyna L, Souhami E, Silvestre $\mathrm{L}$, et al. Adding once-daily lixisenatide for type 2 diabetes inadequately controlled with newly initiated and continuously titrated basal insulin glargine: a 24-week, randomized, placebo-controlled study (GetGoalDuo 1). Diabetes Care 2013; 36: 2497-503.

46. Yoon N, Cavaghan M, Brunelle R, Roach P. Exenatide added to insulin therapy: a retrospective review of clinical practice over two years in an academic endocrinology outpatient setting. Clin Ther 2009; 31: 1511-23.

47. Lind M, Jendle J, Torffvit O, Lager I. Glucagon-like peptide 1 (GLP-1) analogue combined with insulin reduces $\mathrm{HbAlc}$ and weight with low risk of hypoglycemia and high treatment satisfaction. Prim Care Diabetes 2012; 6: 41-6.

48. Lane W, Weinrib S, Rappaport J. The effect of liraglutide added to U-500 insulin in patients with type 2 diabetes and high insulin requirements. Diabet Tech Ther 2011; 13: 592-5.

49. Rosenstock J, Fonseca VA, Gross JL, Ratner RE, Ahrén B, Chow FCC, et al. Advancing basal insulin replacement in type 2 diabetes inadequately 
controlled with insulin glargine plus oral agents: a comparison of adding albiglutide, a weekly GLP-1 receptor agonist, versus thrice-daily prandial insulin lispro. Diabetes Care 2014; 37: 2317-25.

50. Diamant M, Nauck MA, Shaginian R, Malone JK, Cleall S, Reaney M, et al. Glucagon-like peptide 1 receptor agonist or bolus insulin with optimized basal insulin in type 2 diabetes. Diabetes Care 2014; 37: 2763-73.

51. Rosenstock J, Guerci B, Hanefeld M, Gentile S, Aronson R, Tinahones FJ, et al. Prandial options to advance basal insulin glargine therapy: testing lixisenatide plus basal insulin versus insulin glulisine either as basal-plus or basal-bolus in type 2 diabetes: the GetGoal Duo- 2 Trial. Diabetes Care 2016; 39: 1318-28.

52. Mathieu C, Rodbard HW, Cariou B, Handelsman Y, Philis-Tsimikas A, Ocampo Francisco AM, et al. A comparison of adding liraglutide versus a single daily dose of insulin aspart to insulin degludec in subjects with type 2 diabetes (BEGIN: VICTOZA ADD-ON). Diabetes Obes Metab 2014; 16: 636-44.

53. Lingvay I, Pérez Manghi F, García-Hernández P, Norwood P, Lehmann L, Tarp-Johansen MJ, et al. Effect of insulin glargine up-titration vs insulin degludec/liraglutide on glycated hemoglobin levels in patients with uncontrolled type 2 diabetes. JAMA 2016; 315: 898-907.

54. Buse JB, Vilsbøll T, Thurman J, Blevins TC, Langbakke IH, Bøttcher SG, et al. Contribution of liraglutide in the fixed-ratio combination of insulin degludec and liraglutide (IDegLira). Diabetes Care. 2014; 37(11):2926.

55. Freemantle N, Mamdani M, Vilsboll T, Kongsø JH, Kvist K, Bain SC. IDegLira versus alternative intensification strategies in patients with type 2 diabetes inadequately controlled on basal insulin therapy. Diabetes Ther 2015; 6: 573-91.
56. Rosenstock J, Diamant M, Aroda VR, Silvestre L, Souhami E, Zhou T, et al. Efficacy and safety of LixiLan, a titratable fixed-ration combination of lixisenatide and insulin glargine, versus insulin glargine in type 2 diabetes inadequately controlled on metformin monotherapy: the LixiLan proof-of-concept randomized trial. Diabetes Care 2016; 39: 1579-86.

57. Novo Nordisk. FDA extends regulatory review period for IDegLira by three months. (September 2016). Available at: http://www.novonordisk. com/bin/getPDF.2039453.pdf. Accessed on Oct 18, 2016.

58. Vilsbøll T, Vora J, Jarlov H, Kvist K, Blonde L. Type 2 diabetes patients reach target glycemic control faster using IDegLira than either insulin degludec or liraglutide given alone. Clin Drug Investig 2016; 36: 293-303.

59. Watkins E, Kang J, Trautmann M, Choi S, Han O, Hompesch M, et al. LAPS CA-Exendin-4 (efpeglenatide) enhances insulin secretion and beta cell responsiveness in subjects with type 2 diabetes. Poster presented at European Association for the Study of Diabetes (EASD) 51st Annual Meeting. Stockholm, Sweden; 2015 Sept. Available at: http:// www.hanmi.co.kr/hanmi/img/rnd/2015_EASD_(HM11260C).pdf. Accessed on June 4, 2017.

60. Quantum Project. In: Hanmi Pharmaceuticals [online database]. Seoul, Korea. Available at: http://www.hanmipharm.com/ehanmi/handler/ Rnd-ProjectQuantum. Accessed on October 18, 2016.

How to cite this article: St Onge E, Miller S, Clements E, Celauro L, Barnes K. The role of glucagon-like peptide-1 receptor agonists in the treatment of type 2 diabetes. J Transl Intern Med 2017; 5: 79-89. 